\title{
INCLUSIÓN SOCIAL DE LAS PERSONAS CON DISCAPACIDAD: ESTUDIO PILOTO EN EL CONTEXTO LABORAL DEL PAPEL MEDIADOR DE LA AUTODETERMINACIÓN ENTRE EL CONFLICTO DE ROL Y LA INCLUSIÓN SOCIAL
}

Social Inclusion of People with Disability: Pilot Study in the Workplace of the Mediating Role of Self-Determination between Role Conflict and Social Inclusion

\author{
Carmen María Salvador-Ferrer \\ Universidad de Almería \\ cmsalva@ual.es
}

Recepción: 16 de diciembre de 2019

Aceptación definitiva: 6 de julio de 2020

RESUMEN: En este estudio se pretende conocer la calidad de vida que tienen los trabajadores con discapacidad respecto a la inclusión social, así como si los problemas existentes en el trabajo (conflicto de rol) consideran que son determinantes en dicha inclusión. Además, proponemos un modelo donde se plantea que la autodeterminación tiene un efecto mediador entre el conflicto de rol y la inclusión social. El trabajo se ha realizado con un total de 51 trabajadores con discapacidad pertenecientes a un Centro Especial de Empleo que prestan un servicio de limpieza en la Administración Pública. Los resultados indican que los trabajadores valoran la inclusión social y que el conflicto de rol puede afectar a su inclusión social. Además, los hallazgos respaldan el modelo propuesto, ofreciendo una orientación para conseguir la inclusión social del colectivo con discapacidad. Al final planteamos las implicaciones prácticas del presente trabajo y, también, se debate en torno al relevante papel que desempeña el trabajo en la inclusión social.

Palabras Clave: inclusión social; conflicto de rol; autodeterminación; mediación; centro especial de empleo; discapacidad. 
АвsтRAст: This study aims to know the perception that workers with disability have regarding social inclusion, as well as whether the problems at work (role conflict) consider them to be determinants in such inclusion. In addition, we propose a mediation model that states that self-determination has effect between role conflict and social inclusion. The work has been carried out with a total of 51 workers with disability from a Special Employment Center that they perform a cleaning job in the Public Administration. The results indicate that workers value social inclusion and that role conflict can affect their social integration. In addition, the findings support the proposed model, offering guidance to achieve social inclusion of the group with disability. In the end we propose the practical implications of this work and, also, we discuss the relevant role that work plays in social inclusion.

KEY WORDS: social inclusion; role conflict; self-determination; mediation; special employment center; disability.

Agradecimientos: La realización de este trabajo ha sido posible gracias al Proyecto de Intercambio profesores de la UAL y directivos. Además, la autora quiere expresar su especial agradecimiento al Centro Especial de Empleo Verdiblanca por permitir el acceso muestral.

\section{Introducción}

$\mathrm{E}$ L CONCEPTO DE CALIDAD DE VIDA gira en torno a las condiciones individuales y engloba tanto componentes objetivos como subjetivos. En este sentido, este término podría ser considerado como la forma en que cada persona percibe su realidad. Se trata de un constructo multidimensional, donde los diferentes elementos se encuentran influenciados por componentes culturales, sociales e individuales (Schalock y Verdugo, 2006). En este sentido, estos planteamientos conducen, tal como apuntan Schalock y Verdugo (2006), a que el concepto de calidad de vida sitúa a la persona en el eje central del proceso, enfatizando la equidad, el desarrollo personal, la autodeterminación y la inclusión social, siendo los dos últimos términos los referentes del presente trabajo.

La inclusión social de las personas con discapacidad depende en gran medida del éxito en la integración laboral (Gómez y Peñaranda, 2019). El trabajo es fundamental para el bienestar psicológico del individuo, especialmente en el caso de las personas con discapacidad (Dutta, Garvey, Chan, Chou y Ditchman, 2012). Pese a todo, los datos relacionados con el desempleo muestran porcentajes más altos en el colectivo de personas con discapacidad (Burke, Bezyak, Fraser, Pete, Ditchman y Chan, 2013; Turcotte, 2014; Hoque, Bacon y Parr, 2014). El desempleo tiene consecuencias negativas tanto a nivel personal como a nivel económico. En lo concerniente a la situación económica, las personas desempleadas son más vulnerables a vivir en situación de pobreza social (Alcock, 2008). En lo que respecta a la parcela personal, encontramos que las personas desempleadas presentan una baja autoestima y una percepción negativa de bienestar psicológico (Feldman, 1996). 
En la actualidad, se están produciendo cambios sociales que podrían contribuir a modificar el panorama laboral de las personas con discapacidad. Las empresas parece que comienzan a valorar el capital humano de las personas con discapacidad (Gómez y Peñaranda, 2019) y centran el interés en conseguir la inclusión laboral de las personas con discapacidad mediante la adaptación del entorno y la optimización del desempeño (Gómez y Peñaranda, 2019). No obstante, conseguir la plena integración laboral de personas con discapacidad es un gran "reto social" que puede ser abordado desde diferentes perspectivas (Castañeda, Chiappetta, Guerrero y Hernández, 2019).

Primera, analizando los factores externos que facilitan o bloquean la integración laboral del trabajador (por ejemplo, barreras de desplazamiento, etc.). En esta línea de trabajo se enmarca el modelo social, el cual deposita el interés en el estudio de los factores ambientales y las barreras que existen en el contexto laboral (Evans, 2017). Este modelo ofrece una nueva forma de entender la discapacidad, apelando a un constructo social; dicho con otras palabras, se trata de un término condicionado por factores culturales.

Segunda, estudiando los factores que afectan al proceso de contratación de los trabajadores con discapacidad (por ejemplo, estigma social, necesidades específicas, entre otros elementos). En una investigación realizada por Kaye, Jans y Jones (2011), se concluye que las razones percibidas por las que no se contrata o retiene a los trabajadores con discapacidad es por "la falta de conciencia sobre la discapacidad, problemas o preocupación por el coste y temor por la responsabilidad” (p. 526). En términos generales, parece que existe cierta ignorancia sobre las habilidades que tienen las personas con discapacidad (Hoque, Bacon y Parr, 2014). En este sentido, tal como apunta Evans (2017), podríamos percibir la discapacidad como un término "fluido y temporal" (p. 67), es decir, condicionado por el contexto social.

En línea con lo anterior, Gómez y Peñaranda (2019) señalan los factores que limitan la inclusión laboral de las personas con capacidades diferentes, los cuales son de carácter objetivo y subjetivo. Entre los primeros, podríamos destacar el gran desconocimiento que parece existir en torno a las capacidades o habilidades que tienen las personas con discapacidad; el escaso número de programas orientados a la formación y capacitación; y, también, las dificultades encontradas en los accesos, edificios y medios de transporte. Entre los segundos, subrayamos los prejuicios sociales que existen hacia las personas con discapacidad.

Todo lo expuesto previamente nos conduce a que para lograr la integración laboral se requiere tener presentes las necesidades particulares y/o personales con el propósito de adaptar el entorno laboral y conseguir un empoderamiento psicológico en el trabajo (Conger y Kanungo, 1888). Este término hace referencia a una serie de procesos cognitivos y estados psicológicos internos (significado, competencia, autodeterminación e impacto). En este sentido, el empoderamiento psicológico se compone de cuatro elementos fundamentales: significado, entendido como la importancia o valor, según las necesidades del trabajador, que este otorga a las metas y los objetivos organizacionales; competencia, se trata de la creencia que la persona desarrolla sobre su capacidad para realizar las funciones laborales satisfactoriamente; autodeterminación, es decir, la competencia que tiene el empleado para tomar decisiones en su lugar de trabajo; y, finalmente, impacto, 
el grado en que el empleado considera que su trabajo es fundamental para los resultados de la empresa. Pese a todo, para que se produzca el empoderamiento psicológico, es necesario encontrar ciertas condiciones específicas en el escenario comunitario o contexto social-laboral que faciliten la integración laboral.

El empoderamiento o capacitación psicológica no es posible sin que previamente las personas desarrollen la autodeterminación (Wehmeyer, 1996). Dicho con otras palabras, según Wehmeyer (1996), en la medida en que la persona desarrolle la autodeterminación se conseguirán conductas operativas, tales como autonomía, autorregulación, empoderamiento y autorrealización. Por consiguiente, parece que la autodeterminación es fundamental para el desarrollo social y laboral de las personas, especialmente en el caso del colectivo con discapacidad (Wehmeyer, 1998; Wehmeyer, Agran y Hughes, 1998; Palomo y Tamarit, 2000), y esta podría ser considerada como una cuestión de interdependencia social (Tamarit, 2001).

En términos generales, según Wehmeyer (1996), podríamos definir la autodeterminación como "las capacidades y actitudes requeridas para que uno actúe como el principal agente causal en su propia vida, así como para realizar elecciones con respecto a las propias acciones, libre de interferencias o influencias externas indebidas" (p. 24). Se trata, pues, de que las personas con discapacidad tengan conciencia y control sobre su propia vida (Verdugo, 2000). El camino correcto hacia la autodeterminación se logrará si conseguimos que las personas con discapacidad desarrollen conductas más adaptadas a su entorno y si logramos que perciban que tienen un mayor control sobre sí mismos y, también, sobre su propia conducta (Tamarit, 1998). Enmarcados en la Teoría de la Agencia Causal (Shogren, Wehmeyer, Palmer, Forber-Pratt, Little y López, 2015; Mumbardó, Vicente, Giné, Guardia, Raley y Verdugo, 2017), donde se define la autodeterminación como "una característica disposicional que se pone de manifiesto cuando la persona actúa como agente causal de su propia vida” (Shogren et al., 2015, p. 258), existen tres componentes fundamentales (Vicente, Guillén, Gómez, Ibáñez y Sánchez, 2017): (1) volición, es decir, la autonomía; (2) agencialidad, capacidad de regular sus acciones; y (3) creencias sobre las acciones y control ejercido, esto es, los conocimientos sobre las capacidades y habilidades para lograr los objetivos personales.

Extendiendo nuestros planteamientos más allá, la autodeterminación, en el ámbito laboral, podría ser una estrategia de afrontamiento de los conflictos laborales, entre otros, podría tratarse el conflicto de rol. El conflicto de rol podría definirse como la existencia de ideas o expectativas contrarias vinculadas con el rol profesional (Miles, 1977; Van Sell, Brief y Schuler, 1981), este puede encontrarse relacionado bien con las condiciones, los recursos, los objetivos o las autoexpectativas de los trabajadores. Surdez, Magaña y Sandoval (2015) indican que el conflicto de rol afecta al desempeño laboral y, también, podría repercutir en el logro de metas organizacionales. El conflicto de rol en los trabajadores con discapacidad podría surgir por circunstancias laborales que dificultan su desempeño (Surdez et al., 2015). Según Surdez et al. (2015), existen diversos factores organizacionales que son los responsables del conflicto de rol, tales como demandas conflictivas por parte de superiores y subordinados; expectativas divergentes de personas o grupos relacionados con el rol; sobrecarga de trabajo; inadecuada retroalimentación de otros y de las tareas; e insuficiente identidad de la tarea. 
De igual forma, en el caso de las personas con discapacidad podríamos señalar que el conflicto de rol se manifiesta cuando las demandas del trabajo son incompatibles con las actividades y conductas que el trabajador puede realizar, ya que no dispone de los recursos humanos necesarios y/o suficientes (capacidades) para hacerlo. Generalmente, cuando se produce un conflicto de rol, el trabajador experimenta un malestar psicológico que genera insatisfacción y, también, desconfianza en la empresa. El conflicto de rol es un término importante tanto para el bienestar de los empleados como para optimizar los resultados organizacionales. En relación con el mismo, encontramos que son escasos los estudios centrados en trabajadores con discapacidad. A nuestro entender, es necesario conocer si este término es determinante en este colectivo y, además, obtener información detallada sobre la posible repercusión en la inclusión social de los trabajadores con discapacidad. A todo esto tenemos que añadir el escaso número de apoyos empíricos que existen en el contexto español sobre la calidad de vida en el colectivo de personas con discapacidad (Vicente, Mumbardó, Coma, Verdugo y Giné, 2018). Parece, pues, que existen evidencias más que suficientes que respaldan la necesidad de impulsar investigaciones en el colectivo de personas con discapacidad para analizar detalladamente la calidad de vida, así como los componentes principales y la relación que existe entre estos y el ámbito laboral.

Por consiguiente, nuestro objetivo general se centra en estudiar la calidad de vida de los trabajadores con discapacidad, en nuestro caso, y, según la literatura previa, depositamos el interés en la autodeterminación y la inclusión social. Además, intentaremos profundizar en el papel que desempeñan los problemas del entorno laboral (conflicto de rol) sobre su calidad de vida, en concreto, en la inclusión social. En este sentido, se probó un modelo de mediación (ver Figura 1), mediante el cual se comprobó si la autodeterminación tiene un efecto mediador entre el conflicto de rol y la inclusión social.

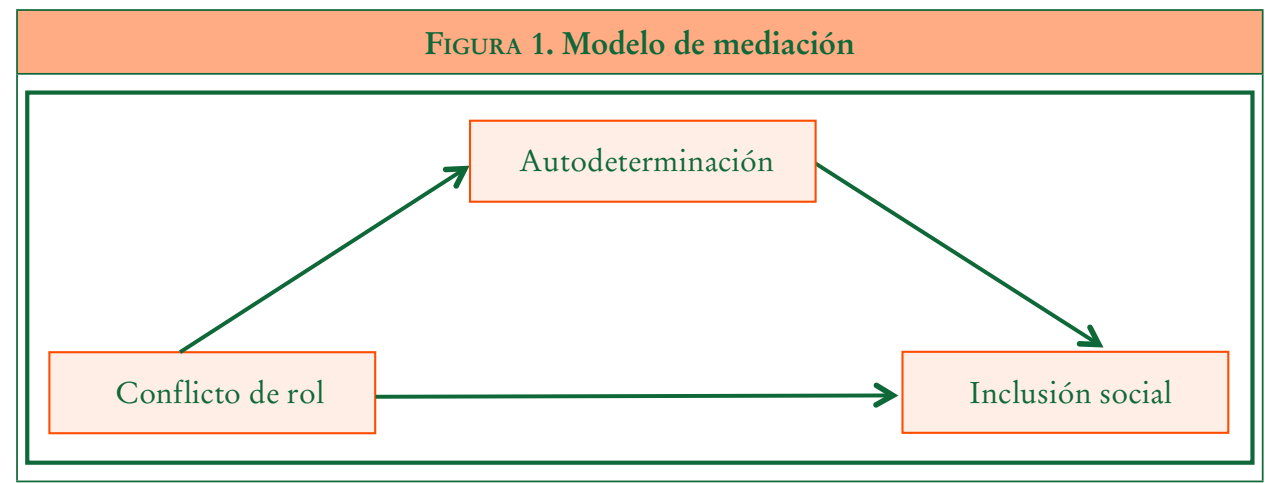

Considerando la literatura previa, nuestros objetivos específicos son los siguientes:

- Primero, conocer la valoración de inclusión social que realizan los trabajadores con discapacidad.

- Segundo, analizar si el conflicto de rol se relaciona significativamente con la inclusión social de los trabajadores con discapacidad. 
INCLUSIÓN SOCIAL DE LAS PERSONAS CON DISCAPACIDAD: ESTUDIO PILOTO EN EL CONTEXTO LABORAL DEL PAPEL MEDIADOR DE LA AUTODETERMINACIÓN ENTRE EL CONFLICTO DE ROL Y LA INCLUSIÓN SOCIAL CARMEN MARÍA SALVADOR-FERRER

- Tercero, proponer y probar un modelo mediante el cual la autodeterminación tiene un efecto mediador entre el conflicto de rol y la inclusión social.

\section{Metodología}

\subsection{Participantes}

El estudio se realizó en un Centro Especial de Empleo con 51 trabajadores que prestan un servicio de limpieza en la Administración Pública (47 \% hombres y 53 \% mujeres), siendo la edad media de 49 años $(S D=9,3)$. En lo concerniente al nivel de estudios, el $50 \%$ con estudios de EGB, seguido del $18 \%$ sin estudios, $18 \%$ Secundaria, $14 \%$ Formación profesional/Bachillerato. En lo relativo al tipo de discapacidad, un $60 \%$ presenta una discapacidad física, un $27 \%$ auditiva, un $7 \%$ con discapacidad psíquica, un $4 \%$ con discapacidad mental y, finalmente, un $2 \%$ con discapacidad visual. En la antigüedad en el puesto, un $49 \%$ cuenta con una experiencia laboral superior a los 10 años, seguido de un $29 \%$ cuya experiencia oscila entre 1 y 5 años, un $16 \%$ lleva menos de un año en el puesto y un $6 \%$ entre 5 y 10 años. Por último, en lo que respecta al estado civil el $57 \%$ está casado/a, seguido de un $17 \%$ soltero/a, un $12 \%$ divorciado/a y/o separado/a, otro $12 \%$ con pareja pero la relación aún no formal y, finalmente, un $2 \%$ viudo/a.

\subsection{Instrumento}

Escala de estrés de rol de Rizzo, House y Lirtzman (1970). En este trabajo utilizamos la escala adaptada al castellano de González-Romá y Llorent (1998). Se trata de un cuestionario, con un formato de respuesta tipo Likert de 7 puntos, siendo 1 "Totalmente en desacuerdo" y 7 “Totalmente de acuerdo", integrado por las siguientes dimensiones: ambigüedad de rol, compuesta por 6 preguntas que hacen referencia a si los trabajadores tienen clara su labor en el puesto de trabajo, un ejemplo de pregunta sería la siguiente: "Yo tengo claro cuál es mi responsabilidad (ítem 3)"; sobrecarga de rol, referida al exceso de trabajo que tiene el empleado, esta dimensión la integran 3 ítems, por ejemplo, "yo tengo mucho trabajo que realizar y poco tiempo (ítem 15)"; y, finalmente, conflicto de rol, referida a existencia de ideas o expectativas contradictorias con el rol profesional, se compone de 8 preguntas (por ejemplo, "yo trabajo en cosas innecesarias -ítem 14-”), nótese que el presente trabajo lo centraremos exclusivamente en el conflicto de rol. El coeficiente alfa de Cronbach de esta escala es de 0,608.

Escala de calidad de vida-FUMAT- de Gómez, Verdugo, Gómez, Arias y Navas (2008). Esta escala ha sido diseñada para evaluar la calidad de vida de cualquier persona usuaria de servicios sociales. En concreto, en este estudio, los participantes eran personas con discapacidad trabajadores de un CEE. Este cuestionario está compuesto por un total de 57 preguntas con formato de respuesta tipo Likert con 4 alternativas, siendo 1 “Totalmente en desacuerdo" y 4 “Totalmente de acuerdo". Según 
Gómez et al. (2009), esta escala se compone de 8 dimensiones, en concreto, las siguientes: bienestar físico, preguntas relacionadas con la salud, la atención sanitaria, el ocio y las actividades diarias; bienestar emocional, asociado con el grado de satisfacción, su percepción de autoconcepto y la ausencia de estrés; relaciones interpersonales, en esta categoría se estudian las interacciones, las relaciones y los apoyos con los que cuenta la persona; inclusión social, es decir, la integración y participación en la comunidad, el papel que la persona percibe que tiene en la comunidad y los apoyos sociales disponibles; desarrollo personal, entendido como la educación, la competencia personal y el desempeño; bienestar material, se analiza el estatus económico, el empleo, la vivienda, etc.; autodeterminación, estudiamos la autonomía, las metas, los valores personales y las elecciones que realiza; y, finalmente, derechos, en concreto, los derechos humanos y legales. En esta investigación se aplicó la escala completa, sin embargo, en el presente artículo nos detendremos solo en el análisis de la autodeterminación y la inclusión social. El coeficiente alfa de Cronbach de esta escala es de 0.857.

\subsection{Procedimiento}

Para la implementación de este trabajo, se llevaron a cabo una serie de gestiones. La primera, se convocaron varias reuniones con la jefa de la Sección de Recursos Humanos del Centro Especial de Empleo. La segunda, la jefa de la sección se reunió con la supervisora de los trabajadores, quien se encargó de realizar varias funciones: a) organizar los grupos, según el turno de trabajo, cada grupo estaba compuesto por un máximo de 10 trabajadores; y b) explicar a los trabajadores el sentido de la tarea y solicitarles su colaboración. La tercera, una vez organizados los grupos, los empleados acudieron, de manera organizada, a la sala de informática de la Universidad de Almería donde se aplicó el cuestionario.

La implementación del cuestionario se llevó a cabo en varios turnos; en función del número de trabajadores de cada turno se realizaron varias sesiones, con una duración máxima de 1 hora. Cuando los empleados se encontraban en la sala, se ofrecía la posibilidad de seleccionar la vía de participación; en este sentido, podían cumplimentar el cuestionario bien en formato digital (google forms) o bien formato físico, siendo la versión física la más demandada. Durante toda la aplicación se encontraba presente una persona, quien se encargaba de aclarar dudas relacionadas con las preguntas. En un plazo de dos semanas se recogieron todos los datos de la presente investigación. A los trabajadores se les pedía su participación voluntaria, asegurándoles el anonimato y la confidencialidad de los datos personales.

\subsection{Análisis de datos}

Para estudiar nuestros objetivos, utilizamos la macro PROCESS (Modelo 4 de mediación simple) para SPSS desarrollada por Hayes (2013). En concreto, realizamos un análisis de mediación simple con una variable mediadora cuantitativa, una muestra de 10000 brootstrapping, modelo 4 y un nivel de confianza del $95 \%$. 


\section{Resultados}

\subsection{Análisis descriptivos}

Dentro de este apartado calculamos las medias, las desviaciones típicas y las correlaciones de Pearson entre las variables del estudio (ver Tabla 1). En lo que respecta a las puntuaciones medias, apreciamos que, en general, los resultados de autodeterminación e inclusión social son superiores al punto medio. El dato más elevado se obtuvo en la escala autodeterminación $(M=3,40, D T=0,50)$, seguido de la inclusión social $(M=3,29, D T=0,540)$. Por su parte, el conflicto de rol obtuvo una puntuación inferior a la puntuación media $(M=2,18, D T=0,85)$.

\begin{tabular}{|l|c|c|c|c|c|}
\hline \multicolumn{6}{|c|}{ TabLa 1. Estadísticos descriptivos y relaciones entre variables } \\
\hline & $M$ & $D T$ & 1 & 2 & 3 \\
\hline 1. Autodeterminación & 3,40 & 0,50 & ----- & & \\
\hline 2. Inclusión social & 3,29 & 0,54 & $0,43 * *$ & ----- & \\
\hline 3. Conflicto de rol & 2,18 & 0,85 & $-0,38 * *$ & $-0,32 *$ & ---- \\
\hline
\end{tabular}

$* \mathrm{p}<0,01 * * * 0,05$

Los resultados del análisis de correlación de Pearson mostraron las relaciones esperadas, existiendo una relación estadísticamente significativa y positiva en el par inclusión social vs. autodeterminación $(r=0,43, p<0,01)$. En el caso del conflicto de rol, observamos que desprende relaciones estadísticamente significativas pero negativas con la autodeterminación y la inclusión social $(r=-0,38, p<0,01 ; r=-0,32, p<0,05$, respectivamente).

\subsection{Análisis de mediación}

Los parámetros generales del modelo indican que los resultados son estadísticamente significativos $(\mathrm{R}=0,45, \mathrm{R}-\mathrm{sq}=0,20, \mathrm{MSE}=0,12, \mathrm{~F}=12,24, p=0,000)$. Los hallazgos obtenidos en el análisis de mediación simple evidencian que la autodeterminación tiene un efecto indirecto $(\beta=0,07 ; \mathrm{SE}=0,04,95 \%$, CI $[0,004,0,160])$ sobre la inclusión social (ver Figura 2).

En primer lugar, los resultados del análisis de regresión lineal simple entre el conflicto de rol y la autodeterminación revelan que el conflicto de rol predice de forma positiva y significativa la autodeterminación $(\beta=0,20 ; t=3,49 ; p<0,001)$.

En segundo lugar, los hallazgos del análisis de regresión múltiple, utilizando como variable predictora el conflicto de rol y la inclusión social, no son estadísticamente significativos $(\beta=0,13 ; t=1,90 ; p<0,06)$, al tiempo que la autodeterminación influye positiva y significativamente en la inclusión social $(\beta=0,35 ; t=2,24 ; p<0,02)$.

En tercer lugar, encontramos que el efecto total del conflicto de rol sobre la inclusión social es estadísticamente significativo $(\beta=0,20 ; t=3,13 ; p<0,002)$. 
En cuarto lugar, en lo que respecta a la relación mediada, apreciamos que existen relaciones positivas y estadísticamente significativas $(\beta=0,07, S E=4 \%, C I[0,004$, $0,16])$. En este sentido, los análisis confirman que el efecto indirecto o de mediación descrito en el modelo es estadísticamente significativo.

En síntesis, tal como muestra la Figura 2, la autodeterminación es una variable mediadora de la relación entre el conflicto de rol y la inclusión social.

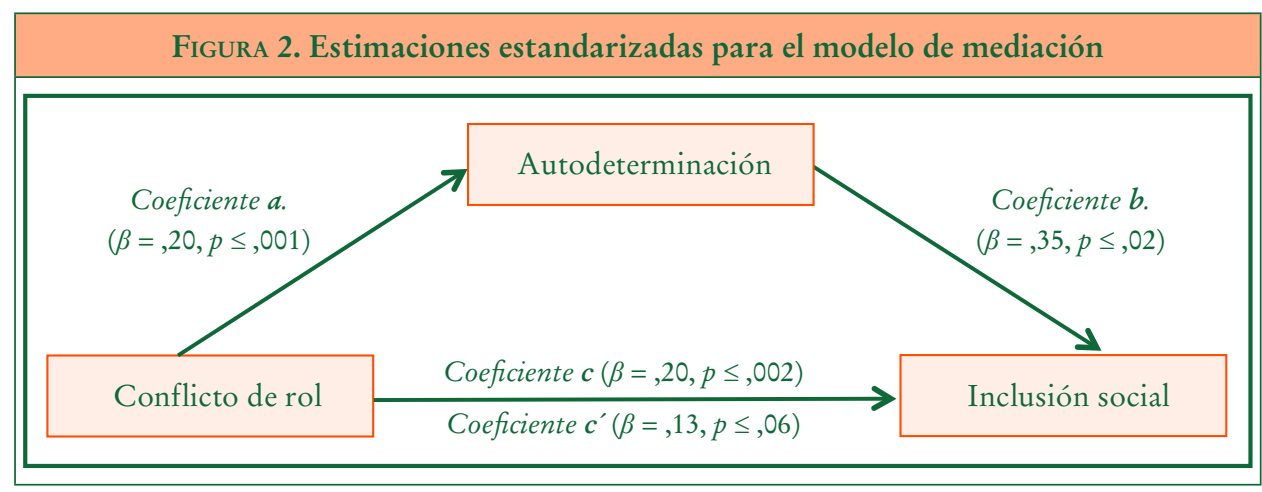

Efecto indirecto (coeficientes $a b$ )

$\beta=, 07, S E=4 \%, C I[, 004,, 16]$

\section{Conclusiones}

Nuestro trabajo se centró en estudiar la calidad de vida de los trabajadores con discapacidad; de manera más detallada, estamos interesados en la inclusión social de los trabajadores con discapacidad, analizando si la existencia de conflictos laborales podría condicionar dicha inclusión. Tal como muestran los datos, el conflicto de rol en el lugar de trabajo podría ser un elemento que dificulte la inclusión social. En concreto, observamos que, aunque los trabajadores señalan no tener conflicto de rol, se observa que existe una relación estadísticamente significativa e inversamente proporcional entre el conflicto de rol y la inclusión social.

En relación con nuestro primer objetivo específico, conocer la valoración de inclusión social que realizan los trabajadores con discapacidad, podríamos señalar que estos trabajadores se sienten incluidos en la sociedad. Tal como indican nuestros datos, la inclusión social es un elemento valorado positivamente por los trabajadores, por lo que podríamos establecer que para las personas con discapacidad la calidad de vida parece estar muy relacionada con la inclusión social (Schalock, 1996).

En lo que respecta al segundo objetivo, analizar si el conflicto de rol se relaciona significativamente con la inclusión social de los trabajadores con discapacidad, destacamos que en función de cómo sea su situación laboral (positiva o negativa) posiblemente así será su grado de inclusión social. En cierto modo, estos datos coinciden con los establecidos por Gómez y Peñaranda (2019) y Gorostidi y Navarro (2007), quienes señalan que el trabajo es un elemento relevante para conseguir la inclusión social. 
Según los datos obtenidos podríamos plantear dos aspectos básicos, el primero, que el trabajo parece tratarse de un elemento importante para lograr la inclusión social y el segundo, hay que conseguir el empoderamiento psicológico laboral no solo para alcanzar mejoras en su lugar de trabajo, sino también para facilitar la integración en la sociedad. Por consiguiente, podríamos plantear que la inclusión social se logra cuando las personas con discapacidad consiguen un empleo ordinario y estable (Gómez y Peñaranda, 2019), adaptado a sus capacidades y habilidades, donde se encuentren perfectamente integrados.

Por ende, las empresas inclusivas deberían extender sus planteamientos más allá de los costes y beneficios, ofreciendo estrategias basadas en la formación y la capacitación de las personas con discapacidad (SOFOFA, 2013), con la finalidad de prevenir la existencia de conflicto de rol en el colectivo de personas con discapacidad. En síntesis, el conflicto de rol podría afectar a la inclusión social; dicho de manera más específica, el trabajo, y cómo sea su experiencia laboral influye en su inclusión social. Este dato encuentra su respaldo en el trabajo de Gómez y Peñaranda (2019), quienes plantean que la inclusión laboral es una herramienta fundamental para lograr la inclusión social.

En lo concerniente al tercer objetivo, proponer y probar un modelo mediante el cual la autodeterminación tiene un efecto mediador entre el conflicto de rol y la inclusión social, descubrimos que la autodeterminación amortigua el conflicto de rol y facilita la inclusión social; dicho con otras palabras, la autodeterminación podría ayudar a superar el conflicto de rol y facilitaría la integración, es decir, la autodeterminación parece que actúa como mediadora entre el conflicto de rol y la inclusión social. Estos resultados llevan a plantear que no hay que compensar los déficits personales, sino más bien invertir en apoyos individualizados para conseguir la participación de la persona en su comunidad, mejorar su calidad de vida y su empoderamiento y autodeterminación (Schalock, 1996). De igual forma, descubrimos que los problemas en el trabajo (conflicto de rol) podrían afectar a su participación social (inclusión social).

Por otro lado, hallamos también que la autodeterminación afecta a la participación o inclusión social (Wehmeyer, 1998; Wehmeyer et al., 1998; Palomo y Tamarit, 2000). Este argumento se sustenta en diferentes investigaciones (Fernández, Morán, Campa, González, Gómez y Monsalve, 2015; Morán, Gómez y Alcedo, 2018), siendo el trabajo de Morán, Gómez y Alcedo (2019) el que respalda principalmente nuestros hallazgos. Estos autores realizan una investigación donde se concluye que en las personas con discapacidad es fundamental trabajar/desarrollar como elementos prioritarios la inclusión social y la autodeterminación.

En lo concerniente a las limitaciones del presente estudio, convendría destacar la dificultad para generalizar los datos, ya que se ha utilizado una muestra muy reducida y el estudio se ha centrado en un servicio concreto. No obstante, a nuestro entender, esta investigación podría suponer un punto de partida para los próximos trabajos, donde se pone de manifiesto la gran importancia que adquiere el entorno laboral y la autodeterminación para el logro de la inclusión social.

En lo que respecta a las implicaciones prácticas del presente trabajo, quisiéramos destacar la relevancia de la autodeterminación y la necesidad de impulsar programas de autodeterminación desde los Centros Educativos (Verdugo, 2000), los Centros 
Especiales de Empleo y las empresas. Tal como apuntan Mumbardó, Guàrdia, Giné, Shogren y Vicente (2018), un aspecto fundamental para lograr la autodeterminación es el proceso educativo psicológico, que consiste en identificar las necesidades específicas en el proceso de toma de decisiones y establecer adaptaciones particulares. Además, a nuestro entender, las empresas inclusivas tienen que invertir esfuerzos para evitar que surjan conflictos de rol, es decir, que las demandas del trabajo no sobrepasen las capacidades de los empleados, ya que, tal como ha quedado patente en nuestro estudio, actuar así facilitaría la inclusión social. En síntesis, nuestros hallazgos revelan la importancia que tiene el trabajo para las personas con discapacidad e invitan a reflexionar sobre la necesidad de realizar acciones de mejora de las condiciones laborales que aseguren su inclusión social.

\section{Referencias bibliográficas}

Alcock, P. (2008). Poverty and social exclusion. En T. Ridge y S. Wright (Eds.), Understanding inequality, poverty and wealth: policies and prospects (pp. 36-60). Chicago, IL: University of Chicago Press.

Burke, J., Bezyak, J., Fraser, R., Pete, J., Ditchman, N. y Chan, F. (2013). Employers' attitudes towards hiring and retaining people with disabilities: a review of the literature. Australian Journal of Rehabilitative Counseling, 19(1), 21-38. https://doi.org/10.1017/ jrc.2013.2

Castañeda, E., Chiappetta, C., Guerrero, L. y Hernández, A. (2019). Empowerment through work: the cases of disabled individuals and low-skilled women workers on the US-Mexican border. Disability and Society, 34(3), 384-406. https://doi.org/10.1080/0968 7599.2018.1545112

Conger, J. A. y Kanungo, R. N. (1988). The empowerment process: integrating theory and practice. The Academy of Management Review, 13(3), 471-482.

Evans, N. J. (2017). Disability in higher education: a social justice approach. San Francisco, CA: Jossey-Bass.

Dutta, A., Garvey, R., Chan, F., Chou, C. y Ditchman, F. (2012). Understanding employers hiring intentions in relation to qualified workers with disabilities: a United States study. Journal of Occupational Rehabilitation, 18, 326-334. http://doi.org/10.1007/s10926009-9220-1

Feldman, D. C. (1996). The nature, antecedents and consequences of underemployment. Journal of Management, 22(3), 385-407. https://doi.org/10.1177/014920639602200302

Fernández, A., Morán, L., Campa, T., González, E., Gómez, L. E. y Monsalve, A. (2015). Resultados personales en autodeterminación en niños y adolescentes con discapacidad intelectual y trastorno de espectro del autismo. En M. Á. Verdugo (Coord.), IX Jornadas cientificas de investigación sobre personas con discapacidad: prácticas profesionales y organizacionales basadas en la evidencia. Salamanca: Inicio.

Gómez, E. y Peñaranda, S. (2019). El estado del arte de la responsabilidad social empresarial e inclusión laboral de las personas con discapacidad. Espacios, 40(22), 19-24.

Gómez, L. E., Verdugo, M. Á., Gómez, B., Arias, P. y Navas, P. (2008). Evaluación de la calidad de vida en personas mayores y con discapacidad: la Escala Fumat. Intervención Psicológica, 17(2), 189-199. 
INCLUSIÓN SOCIAL DE LAS PERSONAS CON DISCAPACIDAD: ESTUDIO PILOTO EN EL CONTEXTO LABORAL DEL PAPEL MEDIADOR DE LA AUTODETERMINACIÓN ENTRE EL CONFLICTO DE ROL Y LA INCLUSIÓN SOCIAL CARMEN MARÍA SALVADOR-FERRER

GonzÁlez-Romá, V. y Lloret, S. (1998). Construct validity of Rizzo et al. (1970) role conflict and ambiguity scales: a multisample study. Applied Psychology: an International Review, 47(4), 535-545. https://doi.org/10.1111/j.1464-0597.1998.tb00042.x

Gorostidi, M. y Navarro, G. (2007). Inserción socio-laboral para los discapacitados. El empleo con apoyo. En J. Ipland et al. (Eds.), Atención a la diversidad. Una responsabilidad compartida. Huelva: Servicio de Publicaciones de Huelva.

Hayes, A. F. (2013). Introduction to mediation, moderation and conditional process analysis. A regression-bassed approach. Nueva York: NY, The Guilford Press.

Hoque, K., BAcon, K. y PARr, D. (2014). Employer disability practice in Britain: assessing the impact of the positive about disabled people 'Two Ticks' Symbol. Work, Employment E Society, 28(3), 430-451.

Kaye, H. S., Jans, L. H. y Jones, E. C. (2011). Why don't employers hire and retain workers with disabilities? Journal of Occupational Rehabilitation, 21(4), 526-536. http://doi. org/10.1007/s10926-011-9302-8

Miles, R. H. (1977). Role-set configuration as a predictor of role conflict and ambiguity in complex organizations. Sociometry, 40(1), 21-34. https://doi.org/10.2307/3033542

Morán, M. L., Gómez, L. y Alcedo, M. A. (2018). 2. Premio de investigación AMPANS 2018. Hacia una mejor calidad de vida en jóvenes con discapacidad intelectual y autismo. Prioridades para la intervención y variables relevantes.

Morán, M. L., Gómez, L. y Alcedo, M. A. (2019). Inclusión social y autodeterminación: los retos en la calidad de vida de los jóvenes con autismo y discapacidad intelectual. Siglo Cero, 50(3), 29-46.

Mumbardó, C., Guàrdia, J., Giné, C., Shogren, K. A. y Vicente, E. (2018). Psychometric properties of the Spanish version of the self-determination inventory student self-report: a structural equation modeling approach. American Journal on Intellectual and Developmental Disabilities, 123(6), 545-557. http://doi.org/10.1352/1944-7558-123.6.545

Mumbardó, C., Vicente, E., Giné, C., Guardia, J., Raley, S. K. y Verdugo, M. Á. (2017). Promoviendo la autodeterminación en el aula: el modelo de enseñanza y aprendizaje de la autodeterminación. Siglo Cero, 48(2), 41-59.

Palomo, R. y Tamarit, J. (2000). Autodeterminación: analizando la elección. Siglo Cero, 189, 21-44.

Rizzo, J., House, R. E. y Lirtzman, J. (1970). Role conflict and ambiguity in complex organization. Administrative Science Quarterly, 15, 150-163. http://dx.doi.org/10.2307/2391486

Schalock, R. L. (1996). Quality of life. Vol. 1: Its conceptualization, measurement and use. Washington, D.C.: American Association on Mental Retardation.

Schalock, R. y Verdugo, M. Á. (2006). El concepto de calidad de vida, su medición y su utilización. En M. Á. Verdugo (Dir.), Cómo mejorar la calidad de vida de las personas con discapacidad. Instrumentos y estrategias de evaluación. Salamanca: Amarú ediciones.

Shogren, K. A., Wehmeyer, M. L., Palmer, S. B., Forber-Pratt, A. J., Little, T. J. y López, S. (2015). Causal Agency Theory: reconceptualizing a functional model of self-determination. Education and Training in Autism and Developmental Disabilities, 50(3), 251-263.

Sociedad de Fomento FABril -SOFOFA-. (2013). Empresa inclusiva. Guía para la contratación de personas con discapacidad. Chile: OIT.

Surdez, E., Magaña, D. y Sandoval, M. C. (2015). Conflicto de rol en profesores universitarios integrantes de cuerpos académicos. Perfiles Educativos, 37(147), 103-125.

TAmarit, J. (1998). Comprensión y tratamiento de conductas desafiantes en las personas con autismo. En A. Rivière y J. MArtos (Comps.), El tratamiento del autismo: nuevas perspectivas. Actas del II Symposium Internacional de Autismo. Madrid: IMSERSO. 
TAmarit, J. (2001). Propuestas para el fomento de la autodeterminación en personas con autismo y retraso mental. En M. Á. Verdugo y F. B. Jordán de Urríes (Eds.), Apoyos, autodeterminación y calidad de vida. Salamanca: Amarú.

Turcotte, M. (2014). Persons with disabilities and employment (Catalogue No. 75-006-X). Statistics Canada. Retrieved April.

VAn Sell, M., Brief, A. P. y Schuler, R. S. (1981). Role conflict and role ambiguity: integration of the literature and directions for future research. Human Relations, I, 43-71. https:// doi.org/10.1177/001872678103400104

Vicente, E., Guillén, V., Gómez, L., Ibáñez, A. y Sánchez, S. (2017). What do stakeholders understand by self-determination? Consensus for its evaluation. Journal of Applied Research in Intellectual Disabilities, 32, 206-218. https://doi.org/10.1111/jar.12523

Vicente, E., Mumbardó, C., Coma, T., Verdugo, M. Á. y Giné, C. (2018). Autodeterminación en personas con discapacidad intelectual y del desarrollo: revisión del concepto, su importancia y retos emergentes. Revista Española de Discapacidad, 6(2), 7-25. http://doi. org/10.5569/2340-5104.06.02.01

Verdugo, M. Á. (2000). Autodeterminación y calidad de vida en alumnos con necesidades educativas especiales. Siglo Cero, 31(3), 5-9.

Wehmeyer, M. L. (1996). Self-determination as an educational outcome. En D. J. SAnds y M. L. Wehmeyer. (Eds.), Self-determination across the life span (pp. 17-36). Baltimore: Paul H. Brookes.

Wehmeyer, M. L. (1998). Self-determination and individual with significant disabilities: examining meaning and misinterpretations. The Journal of the Association for Persons with Severe Handicaps, 25(1), 5-16.

Wehmeyer, M. L., Agran, M. y Hughes, C. (1998). Teaching self-determination to students with disabilities. Baltimore: Paul H. Brookes. 


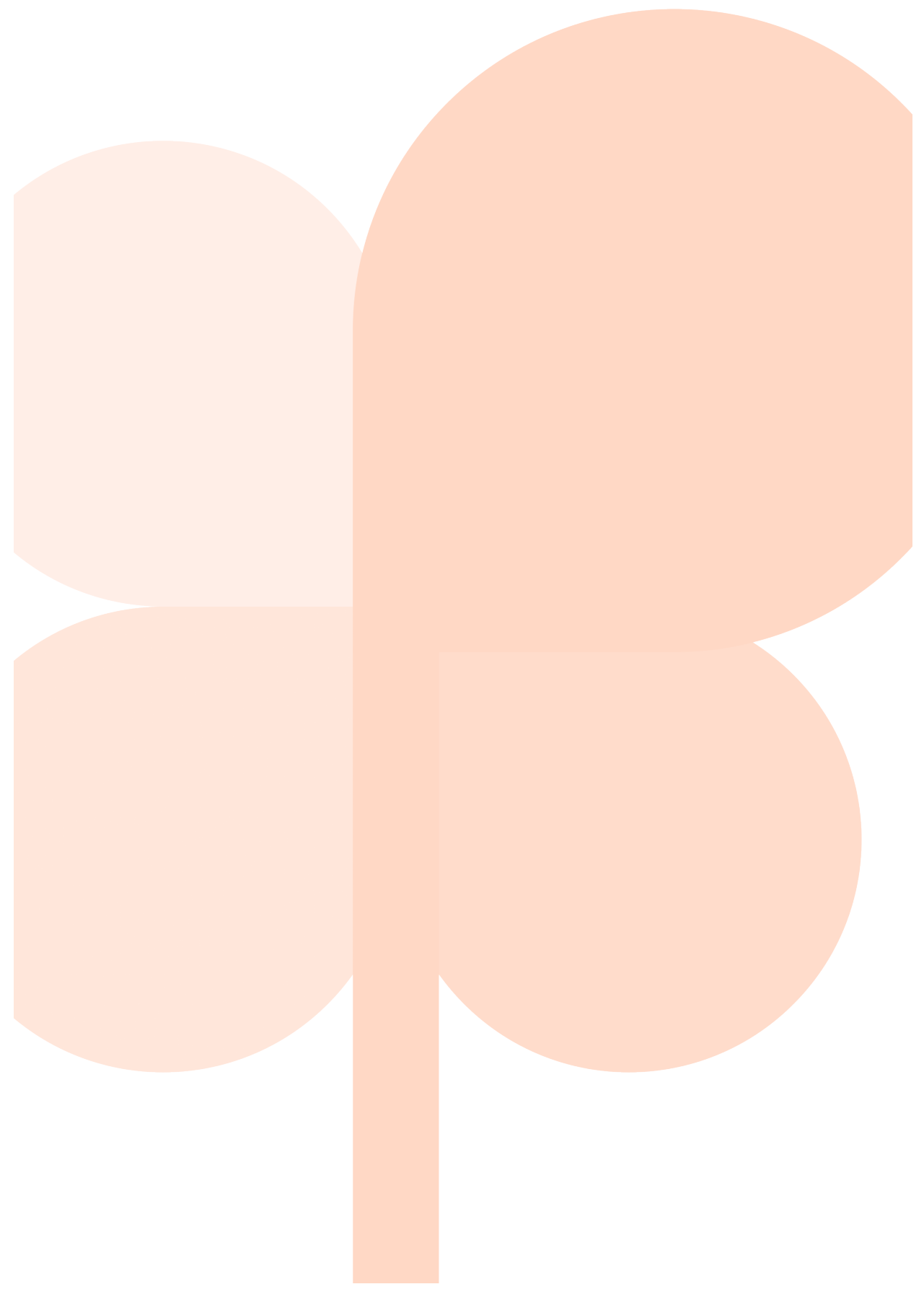

